\title{
Impact of obesity and type 2 diabetes on health-related quality of life in the general population in England
}

This article was published in the following Dove Press journal:

Diabetes, Metabolic Syndrome and Obesity:Targets and Therapy 2 November 2009

Number of times this article has been viewed

\author{
Stephen CL Gough' \\ Nana Kragh ${ }^{2}$ \\ Uffe Jon Ploug ${ }^{2}$ \\ Mette Hammer ${ }^{2}$ \\ 'University of Birmingham and \\ University Hospital Birmingham NHS \\ Foundation Trust, Birmingham, UK; \\ ${ }^{2}$ Novo Nordisk A/S Global Health \\ Economics and Outcomes Research \\ Krogshojvej 55, DK-2880 Bagsvaerd, \\ Denmark
}

Background: Weight gain can contribute towards the development of type 2 diabetes (T2D), and some treatments for T2D can lead to weight gain. The aim of this study was to determine whether having T2D and also being obese had a greater or lesser impact on health-related quality of life (HRQoL) than having either of the two conditions alone.

Methods: The 2003 dataset of the Health Survey for England (HSE) was analyzed using multiple regression analyses to examine the influence of obesity and T2D on HRQoL, and to determine whether there was any interaction between these two disutilities.

Results: T2D reduced HRQoL by 0.029 points, and obesity reduced HRQoL by 0.027 points. There was no significant interaction effect between T2D and obesity, suggesting that the effect of having both T2D and being obese is simply additive and results in a reduction in HRQoL of 0.056 .

Conclusions: Based on analysis of HSE 2003 data, people with either T2D or obesity experience significant reduction in HRQoL and people with both conditions have a reduction in HRQoL equal to the sum of the two independent effects. The effect of obesity on HRQoL in people with T2D should be considered when selecting a therapy.

Keywords: diabetes, health-related quality of life, obesity, type 2 diabetes, England

\section{Background}

Obesity and type 2 diabetes (T2D) are two world health concerns of pressing importance, with the prevalence of both increasing at a startling rate. It is estimated by the International Diabetes Foundation (IDF) that the that the global number of adults with Type 1 diabetes (T1D) or T2D will grow from 248 million in 2007 to 380 million in $2025 .{ }^{1}$ Currently, in developed countries, $85 \%$ to $95 \%$ of people with diabetes have $\mathrm{T} 2 \mathrm{D}$, and in developing countries the proportion with T2D (compared with T1D) is higher. ${ }^{1,2}$ The World Health Organization (WHO) estimated that in 2005 there were more than 400 million obese adults, with body mass index (BMI) $>30 \mathrm{~kg} / \mathrm{m}^{2}$, and 1.6 billion overweight adults. ${ }^{3}$ According to the latest Quality and Outcomes Framework (QOF) estimates, the prevalence of T1D and T2D in England was 3.9\% in 2008 (data for T2D alone are not recorded, but are approximately $90 \%$ of all cases) and the prevalence of obesity was $7.66 \%$ - the prevalence of both are increasing each year. ${ }^{4}$

Both T2D $\mathrm{D}^{5}$ and obesity ${ }^{6}$ reduce HRQoL. The gravest affect of T2D is due to the macro- and micro-vascular complications which usually develop as the disease progresses. ${ }^{7,8}$ Obese people suffer from impaired HRQoL due to specific problems relating to mobility, pain, and/or discomfort, ${ }^{9}$ but also as a result of increased risk of T2D, coronary heart disease (CHD), and hypertension. ${ }^{6,10}$ 
Obesity and T2D are also closely related, since obesity is the largest risk factor for developing T2D. ${ }^{11}$ Therefore, first-line intervention in the management of T2D includes diet and exercise in an attempt to promote weight loss. Unfortunately, however, many of the agents available for the management of T2D often lead to weight gain. ${ }^{12,13}$ Thus knowledge of the relationship between T2D, obesity and HRQoL is desirable.

Several studies have separately investigated weight or diabetes and their effect on HRQoL, but the available literature examining the simultaneous effects of weight and diabetes on HRQoL is limited. ${ }^{14}$ Cross-sectional data are needed to extend the knowledge in this area.

The aim of this study was to evaluate the relationship between obesity, T2D, and HRQoL in a real-world setting in the general population of England.

\section{Methods}

We used data collected during the Health Survey for England (HSE) 2003. ${ }^{15}$ The HSE is a series of annual surveys, commissioned by the UK Department of Health, which covers people living in private households in England. Data collection for HSE 2003 involved an interview, followed by a visit from a specially trained nurse, and included weight and height measurements, as well as further questioning.

Each year the HSE focuses on a different demographic group and looks at health indicators such as cardiovascular disease, physical activity, eating habits, oral health, accidents, and asthma. The 2003 survey had a primary focus on cardiovascular problems and a secondary focus on diabetes. It also included the EQ-5D questionnaire, ${ }^{16}$ a generic health-related quality-of-life measure. The HSE 2003 dataset therefore contained all the parameters for studying the effects of weight, diabetes, confounding illness, and social factors on HRQoL.

The EQ-5D questionnaire is standardized for use as a measure of HRQoL. It provides a simple descriptive profile and a single index value for health status. Each of the five domains consists of three levels (ie, rated by individuals as 'no problems', 'some/moderate problems', or 'extreme/ severe problems'). The five domains of the EQ-5D are: mobility, self-care, usual activity, pain/discomfort, and anxiety/depression. An individual with no problems in any domain would have a score of 1.0. A lower score is recorded if the individual has lower HRQoL, and death is scored as 0.0. Values below zero can exist, if in a given situation, death is considered favorable to life in that given health state. To derive utilities, each health state has been assigned a value based on a UK time-trade-off survey. ${ }^{17}$
During data collection for HSE 2003, participants in the survey were asked if they had diabetes, but not whether they had T1D or T2D. A post-hoc interpretation was applied by those collecting the data, whereby if a participant was receiving insulin at the time of interview, and had been diagnosed with diabetes before their 35th birthday, they would be categorized as having T1D. Otherwise they were categorized as having T2D. Obesity was defined as BMI $\geq 30 \mathrm{~kg} / \mathrm{m}^{2}$ and overweight as BMI of 25 to $29.9 \mathrm{~kg} / \mathrm{m}^{2}$ according to the WHO classification. ${ }^{3,11}$

The original HSE 2003 dataset (18,553 responses) was purged of records from participants that did not receive the EQ-5D questionnaire (those $<16$ years old), or did not complete it; who had T1D; or did not provide measurements needed to calculate BMI. Also discarded were: data from participants who failed to inform about smoking status, cardiovascular disease history, blood pressure, history of long standing, or recent acute illnesses, and participants who did not fill out the General Health Questionnaire (GHQ) (which is designed to predict the need for psychological help). ${ }^{18}$ The GHQ is a validated, self-administered, 12-items questionnaire focusing on the inability to carry out normal functions and the appearance of new and distressing phenomena. ${ }^{19} \mathrm{After}$ purging the dataset, the final number of respondents included in the analyses was 12,188, of which 373 (3.1\%) had T2D.

A multiple linear regression model, consisting of variables describing several factors such as physical and mental well-being, and socio-economic status, and including information on diabetes and BMI, was developed for this study. In the model, HRQoL was dependent on socio-economic and health factors and, for analytical purposes, the focus was on variables for weight and diabetes.

The dataset was analyzed using multiple linear regression analysis to examine the influence of obesity and T2D on HRQoL. Age and gender were retained, even if they became statistically insignificant. Whether or not all other variables were retained depended on the statistical tests. The interaction between the two disutilities, diabetes and obesity, was analyzed to determine whether being obese and having T2D had a further effect beyond the additive effects of having each condition.

The regression model also included variables for socioeconomic status (income), as well as lifestyle indicators affecting health (smoking), and multiple indicators of health status and wellbeing. An acute illness variable described any recent medical history, and a GHQ score measured mental wellbeing. Comorbidity variables contained various long-term medical conditions that could affect HRQoL 
(such as kidney disease, rheumatism, asthma, back problems, bronchitis, cancer, epilepsy, hearing problems, ulcer, cardiovascular disease, and hypertension; Table 2). When testing for statistical significance, some variables were treated as groups (income, smoking, acute illness, and GHQ). Comorbidities were tested individually.

\section{Results}

\section{Demographics}

T2D was primarily present in participants over 40 years of age, and most frequently in people over 60 years (Table 1). Approximately $50 \%$ of people with T2D were also obese, and normal weight individuals had the lowest prevalence of T2D. The trend of higher T2D prevalence with higher BMI group was consistent across age groups, with the exception of the 16-29 year age group, where very small numbers of people with T2D produced inconsistent results, as shown in Table 1.

\section{Regression analysis}

In the model, both T2D and obesity had significant, independent effects on HRQoL, measured by the EQ-5D. Having T2D reduced HRQoL by 0.029 points and obesity reduced HRQoL by 0.027 points. The analysis showed no significant interaction effect between T2D and obesity. The effect of having both conditions is simply the sum of each individual effect (-0.056; Figure 1).

Compared with people whose income was in the lowest fifth of the population, people with higher incomes tended to have a better HRQoL.
Compared with current smokers, people who had stopped smoking, or never started, had a statistically better HRQoL.

Recent suffering from an acute illness, reduced healthrelated HRQoL, and participants who had a high score on the GHQ (indicating mental problems) also had reduced HRQoL. Having other co-morbidities decreased HRQoL, except for hearing problems, which had a positive effect on HRQoL. Also, HRQoL decreased as people got older (Table 2).

\section{Discussion}

This study showed that obese people $\left(\mathrm{BMI} \geq 30 \mathrm{~kg} / \mathrm{m}^{2}\right.$ ) had a lower HRQoL than non-obese people, regardless of whether they have T2D or not. People with T2D, with normal weight (BMI $<25 \mathrm{~kg} / \mathrm{m}^{2}$ ), or obesity, had a lower HRQoL than those in the same weight groups, without the disease. However, the effect of obesity and T2D is purely additive, with no positive or negative effects of having both conditions at the same time.

These results support those from previous investigations of the impact on HRQoL of obesity and T2D. ${ }^{20}$ A study by Macran ${ }^{9}$ that also used HSE data (1996) to examine the relationship between weight (BMI) and HRQoL (with HRQoL quantified according to EQ-5D) found a significant difference in HRQoL between BMI categories, although results differed by gender. For women, but not men, there was a significant decrease in utility scores with increasing BMI, when adjusted for age and longstanding illness. ${ }^{9}$

In a study by Lee et al, ${ }^{21}$ increasing BMI was found to reduce utility in each of the three groups T1D, T2D, and no

Table I Number and proportion of people with type 2 diabetes (T2D) per age and weight group in Health Survey for England 2003

\begin{tabular}{|c|c|c|c|c|c|}
\hline & & BMI $<25 \mathrm{~kg} / \mathrm{m}^{2}$ & BMI $25.0-29.9 \mathrm{~kg} / \mathrm{m}^{2}$ & $\mathrm{BMI} \geq 30 \mathrm{~kg} / \mathrm{m}^{2}$ & All weight groups \\
\hline \multirow[t]{3}{*}{$16-29$ years } & All, $n$ & 1390 & 568 & 266 & 2224 \\
\hline & With T2D, n & 2 & 0 & 3 & 5 \\
\hline & Proportion with T2D, \% & 0.1 & 0.0 & I.I & 0.2 \\
\hline \multirow[t]{3}{*}{$30-49$ years } & All, $n$ & 1780 & 1765 & 1084 & 4629 \\
\hline & With T2D, n & 6 & 14 & 29 & 49 \\
\hline & Proportion with T2D, \% & 0.3 & 0.8 & 2.7 & I.I \\
\hline \multirow[t]{3}{*}{ 50-69 years } & All, $n$ & $107 \mid$ & 1674 & 1073 & 3818 \\
\hline & With T2D, n & 23 & 56 & 108 & 187 \\
\hline & Proportion with T2D, \% & 2.1 & 3.3 & 10.1 & 4.9 \\
\hline \multirow[t]{3}{*}{$70+$ years } & All, $n$ & 456 & 661 & 400 & 1517 \\
\hline & With T2D, n & 23 & 62 & 47 & 132 \\
\hline & Proportion with T2D, \% & 5.0 & 9.4 & 11.8 & 8.7 \\
\hline \multirow[t]{3}{*}{ All ages } & All, $n$ & 4697 & 4668 & 2823 & 12188 \\
\hline & With T2D, n & 54 & 132 & 187 & 373 \\
\hline & Proportion with T2D, \% & I.I & 2.8 & 6.6 & 3.1 \\
\hline
\end{tabular}


Table 2 Overview of results from the regression model

\begin{tabular}{|c|c|c|}
\hline & Parameter estimate & Standard error \\
\hline Intercept & 0.976 & 0.006 \\
\hline Age last birthday & -0.001 & 0.000 \\
\hline Female & -0.005 & 0.003 \\
\hline Diabetes & -0.029 & 0.001 \\
\hline Obese $-\mathrm{BMI}>30$ & -0.027 & 0.003 \\
\hline \multicolumn{3}{|c|}{ Equalized income (as opposed to lowest quintile) } \\
\hline Equalized income - missing & 0.031 & 0.005 \\
\hline Equalized income -2 nd quintile & 0.027 & 0.005 \\
\hline Equalized income - 3rd quintile & 0.040 & 0.005 \\
\hline Equalized income -4 th quintile & 0.056 & 0.005 \\
\hline Equalized income - top quintile & 0.064 & 0.005 \\
\hline \multicolumn{3}{|l|}{ Smoking (as opposed to non-smoking) } \\
\hline Smoking: ex-regular smoker & 0.026 & 0.004 \\
\hline Smoking: never smoker & 0.025 & 0.003 \\
\hline \multicolumn{3}{|c|}{ Acute illness (as opposed to not having had acute } \\
\hline \multicolumn{3}{|c|}{ illness in the last 2 weeks) } \\
\hline Acute illness: I-3 days & -0.039 & 0.007 \\
\hline Acute illness: $4-6$ days & -0.077 & 0.009 \\
\hline Acute illness: $7-13$ days & -0.088 & 0.008 \\
\hline Acute illness: a full 2 weeks & -0.153 & 0.007 \\
\hline \multicolumn{3}{|c|}{ GHQ (as opposed to having a score of zero) } \\
\hline GHQ: score I-3 & -0.058 & 0.003 \\
\hline GHQ: score 4+ & -0.194 & 0.005 \\
\hline \multicolumn{3}{|l|}{ Comorbidities } \\
\hline Kidneys & -0.063 & 0.016 \\
\hline Rheumatism & -0.169 & 0.005 \\
\hline Asthma & -0.013 & 0.006 \\
\hline Back problems & -0.137 & 0.006 \\
\hline Bronchitis & -0.130 & 0.015 \\
\hline Cancer & -0.073 & 0.011 \\
\hline Epilepsy & -0.080 & 0.016 \\
\hline Hearing problems & 0.028 & 0.012 \\
\hline Ulcer & -0.059 & 0.011 \\
\hline CVD (angina, heart attack or stroke) & -0.048 & 0.006 \\
\hline High blood pressure & -0.017 & 0.004 \\
\hline
\end{tabular}

Notes: Values in bold are statistically insignificant (ie, all values were significant $[P<0.05]$ except those for female gender).

Abbreviations: BMI, body mass index; CVD, cardiovascular disease; GHQ, General Health Questionnaire.

diabetes. The effect of both BMI and diabetes on utility was significant. There was no significant difference in the effect of obesity on utility between those with and without diabetes.

A recent review of 18 articles investigating the impact of change in body weight on people's utility scores found that utility decreased as body weight increased, regardless of the scoring system used, or the population. ${ }^{14}$ The review also found that the change in utility score per unit change in BMI, was slightly higher in people with diabetes than in people without diabetes. ${ }^{14}$ Our results support the findings of Matza et al, ${ }^{22}$ who showed that a $3 \%$ higher weight (as a treatment-related attribute) was accompanied by a reduction in HRQoL of 0.04 points.

The reduction in HRQoL associated with having T2D and obesity shown in this study was similar to the impact of aging several decades, or going from having an income in the second highest quintile to being in the lowest quintile. The impact was slightly lower than the impact of a foot ulcer and slightly greater than the impact of a cardiovascular event. 


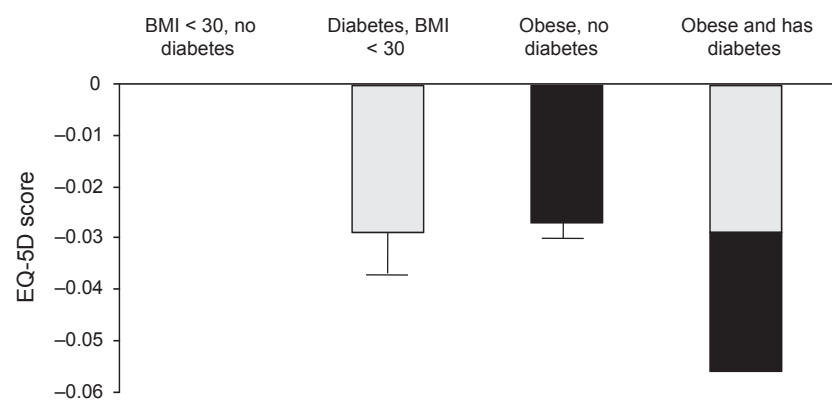

Figure I Effect of type 2 diabetes and obesity on health-related quality of life measured by EQ-5D.

Notes: Mean values and SEM. Error bars not shown for combined (summed) data.

Although neither T2D nor obesity was correlated with an increase in the risk of problems in the EQ-5D sub-model on anxiety (results not shown), people with a high GHQ score (ie, people who reported a need for psychological help), had lower HRQoL. GHQ score could to some extent, be seen as a proxy for anxiety issues stemming from other sources, such as diabetes and obesity. This would support results from the Diabetes Attitudes Wishes and Needs (DAWN) study, in which people reported they would feel a sense of self-blame if they had to start insulin therapy. ${ }^{23}$ The IDF currently recommends that well-being and psychological status are periodically tested in people with T2D, either by questioning or using a formal instrument. ${ }^{24}$

The effect of obesity on HRQoL in people with T2D should be considered when selecting a therapy. Where possible, preference should be given to therapeutic interventions that have a minimal impact on weight gain. In the United Kingdom Prospective Diabetes Study (UKPDS), weight gain was found to be significantly higher in people with T2D receiving intensive treatment than in those receiving conventional treatment. ${ }^{25}$ It is recognized that different treatments can have different effects in weight gain - biguanides, $\alpha$-glucosidase inhibitors, GLP-1 agents, and some insulin analogues are the least likely to cause weight gain and may be associated with weight loss. ${ }^{13,26}$ The results of the multinational DAWN study ${ }^{27}$ show that that one of the priorities among patients with T2D was the avoidance of weight gain.

People with T1D made up almost a quarter of people with diabetes in HSE 2003, compared with the European norm of $4 \%$ to $5 \%,{ }^{28}$ indicating that the interpretation may have classified too many people as having T1D. However, any choice of age limit as a means of distinguishing between diabetes types will tend to be arbitrary and some degree of misclassification is unavoidable. For the purpose of this article the chosen standard for the HSE 2003 was used, but we noted the potential for misclassification and acknowledge that this is one of the limitations of the analysis. However, we do not believe that any misclassification would have had a substantial impact on the overall findings of this analysis.

The HSE dataset analyzed in the current study did not include people in institutions, which is likely to have resulted in a relatively lower representation of the older population, particularly those with disabilities and severe illness. Noninclusion of this population in our assessment could have led to an underestimation of the mean impact on HRQoL of both obesity and diabetes.

The prevalence of T2D was only $3.1 \%$ in the purged dataset, compared with $3.9 \%$ in the UK population in $2003 .{ }^{1}$ However, it was considered that the remaining people with diabetes were representative of the group as a whole, and therefore, the selection bias would not exaggerate the results in the model. Similarly, approximately $34 \%$ of the HSE 2003 population was excluded, introducing the possibility of non-response bias. However, most people excluded from the analysis were children $(<16$ years old; $58 \%$ of those excluded) who did not complete the EQ-5D and would not have been classified as T2D patients in our analysis.

\section{Conclusions}

The results of this study of data from HSE 2003 show that having T2D and being obese, as individual conditions, reduce HRQoL significantly, but that the effect of having both conditions is purely additive. When we consider that many treatments for T2D cause weight gain, the effect of a treatment-related increase in weight on the HRQoL of people with T2D should be taken into account when choosing treatment. In future, longitudinal data may provide information on the effect on HRQoL of treatment-associated weight gain on people with T2D.

\section{Authors' contributions}

SG contributed to the conception and design of the study, the interpretation of the data and critical revision of the manuscript. NK, UJP and MH contributed to the conception and design of the study, data acquisition, data analysis and interpretation and critical revision of the manuscript. All authors read and approved the final manuscript.

\section{Acknowledgments}

This study was sponsored by Novo Nordisk A/S. The Health Survey for England 2003 was carried out by the Joint Health Surveys Unit (University College London and the National Centre for Social Research). The Health Survey for England 
was funded by the Department of Health. Editorial assistance was provided by ESP Bioscience (Sandhurst, UK) on behalf of Novo Nordisk A/S.

\section{Disclosures}

SG has received funds for research, a fee for speaking and fees for consulting from Novo Nordisk. NK, UJP and $\mathrm{MH}$ are all employees of Novo Nordisk A/S.

\section{References}

1. Chapter 1. In: Diabetes Atlas, 3rd ed. International Diabetes Federation; 2006:19.

2. The global burden of diabetes. In: Diabetes Atlas, 2nd ed. International Diabetes Federation; 2003:15-111.

3. WHO fact sheet (No. 311) on obesity and overweight. URL: http:// www.who.int/mediacentre/factsheets/fs311/en/index.html. Accessed 4 September 2009.

4. QOF Reports. Diabetes p-2008. URL: http://www.diabetes.org.uk/ Professionals/Information_resources/Reports/Diabetes-prevalence2008/. Accessed 4 September 2009.

5. Rubin RR, Peyrot M. Quality of life and diabetes. Diabetes Metab Res Rev. 1999;15:205-218.

6. Kolotkin RL, Crosby RD, Williams GR, Hartley GG, Nicol S. The relationship between health-related quality of life and weight loss. Obesity Res. 2001;9:564-571.

7. Graue M, Wentzel-Larsen T, Hanestad BR, Batsvik B, Sovik O. Measuring self-reported health-related quality of life in adolescent with type 1 diabetes using both generic and disease specific instruments. Acta Paediatr. 2003;92:1190-1196.

8. Coffey JT, Brandle M, Zhou H, et al. Valuing health-related quality of life in diabetes. Diabetes Care. 2002;25:2238-2243.

9. Macran S. The relationship between body mass index and health-related quality of life. Centre for Health Economics, University of York; 2004: Discussion paper 190.

10. Jia H, Lubetkin EI. The impact of obesity on health-related qualityof-life in the general adult US population. J Public Health (Oxf). 2005;27:156-164.

11. James WPT, Jackson-Leach R, Mhurdu CN, et al. Overweight and obesity. In: Ezzati M, Lopez, AD, Rodgers A, Murray CJL. Eds. Comparative Quantification of Health Risks: Global and Regional Burden of Disease Attributable to Selected Major Risk Factors. WHO; 2003.

12. AACE/ACE: Medical Guidelines for Clinical Practice for the Management of Diabetes Mellitus. Endocr Pract. 2007; 13 Suppl 1:3-68.
13. Hermansen K, Mortensen LS. Bodyweight changes associated with antihyperglycaemic agents in type 2 diabetes mellitus. Drug Saf. 2007;30:1127-1142.

14. Dennett SL, Boye KS, Yurgin NR. The impact of body weight on patient utilities with or without Type 2 diabetes: a review of the medical literature. Value Health. 2008;11:478-486.

15. Health Survey for England 2003. URL: http://www.dh.gov.uk/en/ Publicationsandstatistics/Publications/PublicationsStatistics/DH_ 4098712. Accessed 07 February 2007.

16. The Euroqol Group: Euroqol - a new facility for the measurement of health-related quality of life. Health Policy. 1990;16:199-208.

17. Dolan P, Gudex C, Kind P, Williams A. A social tariff for EuroQol: results from a UK general population survey. Centre for Health Economics, University of York. 1995: Discussion paper 138.

18. Goldberg D, Williams PA. User's Guide to the General Health Questionnaire. Windsor, UK, NFER-Nelson. 1998.

19. Pevalin DJ. Multiple applications of the GHQ-12 in a general population sample: an investigation of long-term retest effects. Soc Psychiatry Psychiatr Epidemiology. 2000;35:508-512.

20. Bradley C, Speight J. Patient perceptions of diabetes therapy: assessing quality of life. Diabetes Metab Res Rev. 2002;18:S64-S69.

21. Lee AJ, Morgan CLI, Morissey M, Wittrup-Jensen KU, Kennedy-Martin T, Currie CJ. Evaluation of the association between EQ-5D index (healthrelated utility) and body mass index (obesity) in hospital-treated people with type 1 diabetes, type 2 diabetes and no diagnosed diabetes. Diabet Med. 2005;22:1482-1486.

22. Matza LS, Boye KS, Yurgin N, et al. Utilities and disutilities for type 2 diabetes treatment-related attributes. Qual Life Res. 2007;16: 1251-1265.

23. Peyrot M, Rubin RR, Lauritzen T, et al. Resistance to insulin therapy among patients and providers. Results of the cross-national Diabetes Attitudes, Wishes, and Needs (DAWN) study. Diabetes Care. 2005; 28:2673-2679.

24. IDF Clinical Guidelines Task Force: Chapter 4, Psychological care. Global Guideline for Type 2 Diabetes. 2005:19-21.

25. UK Prospective Diabetes Study (UKPDS) Group. Intensive blood-glucose control with sulphonylureas or insulin compared with conventional treatment and risk of complications in patients with type 2 diabetes (UKPDS 33). Lancet. 1998;352:837-853.

26. Nathan DM, Buse JB, Davidson MB, et al. Medical management of hyperglycemia in type 2 diabetes: a consensus algorithm for the initiation and adjustment of therapy. Diabetes Care. 2009;32:193-203.

27. Rubin RR, Peyrot M, Siminerio LM. Health care and patient-reported outcomes: results of the cross-national Diabetes Attitudes, Wishes and Needs (DAWN) study. Diabetes Care. 2006;29:1249-1255.

28. Amos AF, McCarty DJ, Zimmet P. The rising global burden of diabetes and its complications: estimates and projections to the year 2010. Diabet Med. 1997; 14:S7-S85.

\section{Publish your work in this journal}

Diabetes, Metabolic Syndrome and Obesity: Targets and Therapy is an international, peer-reviewed open-access journal committed to the rapid publication of the latest laboratory and clinical findings in the fields of diabetes, metabolic syndrome and obesity research. Original research, review, case reports, hypothesis formation, expert opinion and commentaries are all considered for publication. The manuscript management system is completely online and includes a very quick and fair peer-review system, which is all easy to use. Visit http://www. dovepress.com/testimonials.php to read real quotes from published authors. 